\title{
First Principle Gas Phase Study of the Trans and Gauche Rotamers of 1,2-Diisocyanoethane, 1,2-Diisocyanodisilane and Isocyano(isocyanomethyl)silane
}

\author{
Ponnadurai Ramasami \\ Department of Chemistry, University of Mauritius, \\ Réduit, Republic of Mauritius \\ p.ramasami@uom.ac.mu
}

\begin{abstract}
The trans and gauche rotamers of 1,2-diisocyanoethane, 1,2diisocyanodisilane and isocyano(isocyanomethyl)silane have been studied in the gas phase. A transition state has also been obtained for the interconversion of these rotamers. The methods used are MP2 and DFT/B3LYP and the basis sets used for all atoms are 6-311++G(d,p). The optimised geometries, dipole moments, moment of inertia, energies, energy differences and rotational barriers are reported. Vibrational frequencies of the rotamers are presented with appropriate assignments. The results indicate that the trans rotamer is more stable and the G2MP2 rotational energy differences are $2.97 \mathrm{~kJ} / \mathrm{mol}$ (1,2-diisocyanoethane), $3.02 \mathrm{~kJ} / \mathrm{mol}$ (isocyano(isocyanomethyl)silane) and $2.12 \mathrm{~kJ} / \mathrm{mol}$ (1,2-diisocyanodisilane). The rotational barrier for 1,2-diisocyanoethane is larger than its energy difference but the barrier becomes comparable to the energy difference for isocyano(isocyanomethyl)silane.
\end{abstract}

Keywords: 1, 2-diisocyanoethane, 1, 2-diisocyanodisilane and isocyano (isocyanomethyl)silane, MP2, DFT/B3LYP, energy difference, rotational barrier.

\section{Introduction}

1,2-Diisocyanoethane is a symmetrical 1,2-disubstituted ethane and therefore exists as two rotamers namely the trans and gauche rotamers which are in equilibrium [1-5]. 1,2-Dicyanoethane has been the subject of experimental [6] and theoretical conformational studies [7-11]. However 1,2-diisocyanoethane has not been comparably studied although the isocyano group is one of the most polar neutral substituents.

Schrumpf and Martin [12] reported the conformation and vibrational spectra of 1,2-diisocyanoethane by measuring its infrared spectra in the vapour, liquid crystalline solid and several solvents. They also carried out semiempirical computations at the INDO level and concluded that the two forms are of about equal stabilities with the gauche form being slightly favoured. However they pointed about the shortcomings of semi empirical computation, which can be remedied by more refined ab initio level. Schrumpf et al. [13] reported the molecular structure of 1,2-diisocyanoethane obtained using the gas electron-diffraction method. They identified two distinct rotamers namely the trans and gauche forms. They concluded that the trans form is 
more stable with a percentage of 56.9 in the equilibrium mixture. In a previous communication [11], a theoretical gas phase study of trans and gauche rotamers of 1,2dicyanoethane and structurally analogous compounds, 1,2-dicyanodisilane and cyano(cyanomethyl)silane, was reported in terms of molecular structures, energies and infrared frequencies. The results indicate that in general, the energy difference between the trans and gauche rotamers is in the order: 1,2-dicyanoethane > cyano(cyanomethyl)silane > 1,2-dicyanodisilane.

Thus although 1,2-diisocyanoethane has received attention, to the best of our knowledge there have not been studies involving 1,2-diisocyanodisilane and isocyano(isocyanomethyl)silane although they are structurally related to 1,2diisocyanoethane. Some of the motivations of this work are derived from the limited literature of 1,2-diisocyanodisilane and isocyano(isocyanomethyl)silane and more importantly, isocyano compounds are widely used as ligands [14] and occur naturally in marine organisms [15].

In this paper, the molecular structures, energy difference $(\Delta \mathrm{E})$ between the gauche and trans rotamers, rotational barrier, rotational thermodynamics, and vibrational spectra of the trans and gauche rotamers have been obtained for the title compounds using MP2 and DFT/B3LYP methods. Some of the results obtained are compared with the reported parameters of their isomeric dicyano compounds [11]. The findings of this work are hereby reported.

\section{Methods}

MP2 and DFT/B3LYP computations have been carried out for molecular geometry optimisation of the trans and gauche rotamers of the title compounds. The trans rotamers of 1,2-diisocyanoethane and 1,2-diisocyanodisilane have been studied in $C_{2 \mathrm{~h}}$ symmetry but the trans rotamer of isocyano(isocyanomethyl)silane has been considered in $C_{\mathrm{s}}$ symmetry. The gauche rotamers of 1,2-diisocyanoethane and 1,2diisocyanodisilane have been studied in $C_{2}$ symmetry but the gauche rotamer of isocyano(isocyanomethyl)silane has been considered in $C_{1}$ symmetry. A transition state has also been obtained for the interconversion of these rotamers. The basis sets used for all atoms are 6-311++G(d,p). G2/MP2 computations have also been carried out. Frequency computations have been carried out using the optimised structures. All computations have been done using Gaussian 03W [16] program suite and Gauss View [17] has been used for visualising the rotamers.

\section{Results and Discussion}

The relevant structural optimised structures of the trans and gauche rotamers of the title compounds are reported in Table 1. Several conclusions can be drawn from Table 1. Firstly, there is little difference between the values of the different parameters obtained using the two methods of theory. Secondly, there is a good comparison 
between some of the calculated parameters and available literature data 1,2diisocyanoethane. To be more precise, the reported bond lengths $(\AA)$ of $\mathrm{C}-\mathrm{H}, \mathrm{N} \equiv \mathrm{C}, \mathrm{C}$ $\mathrm{C}$ and $\mathrm{C}-\mathrm{N}$ are 1.123. 1.172, 1.529 and 1.424 respectively [13]. Further the torsional angle $\mathrm{N}-\mathrm{C}-\mathrm{C}-\mathrm{N}$ for the gauche conformer is reported to be $56.9^{\circ}$ [13]. Thirdly, the torsional angles calculated for the gauche rotamers are generally greater from DFT than MP2 computations. Lastly, the moment of inertias calculated for the rotamers follow the order $\mathrm{I}_{\mathrm{A}} \gg \mathrm{I}_{\mathrm{B}} \approx \mathrm{I}_{\mathrm{C}}$. Apart from these, the effect of substituting the cyano group by the isocyano group can be found by comparing the results obtained with those reported [11]. It is found that substitution affects mainly the bond length between carbon and nitrogen with the bond being shorter in case of the dicyano compounds. The transition state modeled corresponds to the isocyano group eclipsing

Table 1. Optimised parameters of the trans and gauche rotamers of 1,2-diisocyanoethane, 1,2diisocyanodisilane and isocyano(isocyanomethyl)silane

\begin{tabular}{|c|c|c|c|c|}
\hline & \multicolumn{2}{|c|}{ MP2 } & \multicolumn{2}{|c|}{ DFT } \\
\hline & \multicolumn{4}{|c|}{$\mathrm{CNCH}_{2} \mathrm{CH}_{2} \mathrm{NC}$} \\
\hline Parameter & Trans & Gauche & Trans & Gauche \\
\hline $\mathrm{r}(\mathrm{C}-\mathrm{H}) / \AA$ & 1.092 & 1.093 & 1.092 & 1.093 \\
\hline $\mathrm{r}(\mathrm{N} \equiv \mathrm{C}) / \AA$ & 1.186 & 1.187 & 1.171 & 1.171 \\
\hline $\mathrm{r}(\mathrm{C}-\mathrm{C}) / \AA$ & 1.534 & 1.529 & 1.540 & 1.535 \\
\hline $\mathrm{r}(\mathrm{C}-\mathrm{N}) / \AA$ & 1.423 & 1.423 & 1.423 & 1.422 \\
\hline$\tau(\mathrm{N}-\mathrm{C}-\mathrm{C}-\mathrm{N}) /^{\circ}$ & 180.0 & 64.2 & 180.0 & 67.6 \\
\hline$\mu /$ Debye & 0 & 5.239 & 0 & 5.554 \\
\hline $\mathrm{I}_{\mathrm{A}} / \mathrm{GHz}$ & 24.689 & 7.110 & 25.465 & 7.579 \\
\hline $\mathrm{I}_{\mathrm{B}} / \mathrm{GHz}$ & 1.649 & 2.690 & 1.633 & 2.508 \\
\hline \multirow[t]{2}{*}{$\mathrm{I}_{\mathrm{C}} / \mathrm{GHz}$} & 1.576 & 2.110 & 1.564 & 2.029 \\
\hline & \multicolumn{4}{|c|}{$\mathrm{CNSiH}_{2} \mathrm{SiH}_{2} \mathrm{NC}$} \\
\hline $\mathrm{r}(\mathrm{Si}-\mathrm{H}) / \AA$ & 1.470 & 1.472 & 1.479 & 1.479 \\
\hline $\mathrm{r}(\mathrm{N} \equiv \mathrm{C}) / \AA$ & 1.191 & 1.191 & 1.178 & 1.178 \\
\hline $\mathrm{r}(\mathrm{Si}-\mathrm{Si}) / \AA$ & 2.349 & 2.348 & 2.360 & 2.360 \\
\hline $\mathrm{r}(\mathrm{Si}-\mathrm{N}) / \AA$ & 1.762 & 1.758 & 1.758 & 1.754 \\
\hline$\tau(\mathrm{N}-\mathrm{Si}-\mathrm{Si}-\mathrm{N}) /^{\circ}$ & 180.0 & 64.1 & 180.0 & 69.5 \\
\hline$\mu$ / Debye & 0 & 4.584 & 0 & 4.836 \\
\hline $\mathrm{I}_{\mathrm{A}} / \mathrm{GHz}$ & 7.647 & 3.441 & 7.833 & 3.716 \\
\hline $\mathrm{I}_{\mathrm{B}} / \mathrm{GHz}$ & 1.059 & 1.494 & 1.045 & 1.360 \\
\hline \multirow[t]{2}{*}{$\mathrm{I}_{\mathrm{C}} / \mathrm{GHz}$} & 0.951 & 1.186 & 0.942 & 1.126 \\
\hline & \multicolumn{4}{|c|}{$\mathrm{CNSiH}_{2} \mathrm{CH}_{2} \mathrm{NC}$} \\
\hline $\mathrm{r}(\mathrm{Si}-\mathrm{H}) / \AA$ & 1.446 & 1.469 & 1.475 & 1.478 \\
\hline $\mathrm{r}(\mathrm{C}-\mathrm{H}) / \AA$ & 1.094 & 1.095 & 1.094 & 1.095 \\
\hline $\mathrm{r}\left({ }_{\mathrm{C}} \mathrm{N} \equiv \mathrm{C}\right) / \AA$ & 1.187 & 1.187 & 1.172 & 1.172 \\
\hline $\mathrm{r}\left(\mathrm{si}_{\mathrm{i}} \mathrm{N} \equiv \mathrm{C}\right) / \AA$ & 1.191 & 1.190 & 1.178 & 1.178 \\
\hline $\mathrm{r}(\mathrm{Si}-\mathrm{C}) / \AA$ & 1.894 & 1.893 & 1.905 & 1.903 \\
\hline $\mathrm{r}(\mathrm{Si}-\mathrm{N}) / \AA$ & 1.756 & 1.750 & 1.753 & 1.748 \\
\hline $\mathrm{r}(\mathrm{C}-\mathrm{N}) / \AA$ & 1.426 & 1.424 & 1.424 & 1.421 \\
\hline$\tau(\mathrm{N}-\mathrm{Si}-\mathrm{C}-\mathrm{N}) /^{\circ}$ & 180.0 & 63.9 & 180.0 & 68.0 \\
\hline$\mu /$ Debye & 0.642 & 6.192 & 0.451 & 5.236 \\
\hline $\mathrm{I}_{\mathrm{A}} / \mathrm{GHz}$ & 12.994 & 4.299 & 13.273 & 5.028 \\
\hline $\mathrm{I}_{\mathrm{B}} / \mathrm{GHz}$ & 1.347 & 1.900 & 1.331 & 1.911 \\
\hline $\mathrm{I}_{\mathrm{C}} / \mathrm{GHz}$ & 1.248 & 1.440 & 1.237 & 1.517 \\
\hline
\end{tabular}


Table 2. Energies of the trans and gauche rotamers of 1,2-diisocyanoethane, 1,2diisocyanodisilane and isocyano(isocyanomethyl)silane

\begin{tabular}{|c|c|c|c|c|c|c|c|}
\hline & & & $\Delta \mathrm{E} / \mathrm{kJ} / \mathrm{mol}$ & $\begin{array}{r}\Delta \mathrm{H} / \\
\mathrm{kJ} / \mathrm{mol}\end{array}$ & $\begin{array}{r}\Delta \mathrm{H} / \\
\mathrm{kJ} / \mathrm{mol}\end{array}$ & $\begin{array}{r}\Delta \mathrm{G} / \\
\mathrm{kJ} / \mathrm{mol}\end{array}$ & $\begin{array}{r}\Delta \mathrm{S} \\
\mathrm{J} / \mathrm{mol}\end{array}$ \\
\hline \multicolumn{8}{|c|}{ 1,2-Diisocyanoethane } \\
\hline $\begin{array}{l}\text { DFT/B3LYP/ } \\
6-311++\mathrm{G}(\mathrm{d}, \mathrm{p})\end{array}$ & $\begin{array}{c}-264.308002 \\
(0.072546)\end{array}$ & $\begin{array}{c}-264.306678 \\
(0.072599)\end{array}$ & 3.48 & & 3.33 & $\begin{array}{c}3.50 \\
(67.2)\end{array}$ & 0.55 \\
\hline G2MP2 & $\begin{array}{c}-263.785502 \\
(0.070277)\end{array}$ & $\begin{array}{c}-263.784369 \\
(0.070364)\end{array}$ & & 2.97 & 2.70 & $\begin{array}{c}2.84 \\
(61.1)\end{array}$ & 0.48 \\
\hline \multicolumn{8}{|c|}{ 1,2-Diisocyanodisilane } \\
\hline MP2/6-311++G(d,p) & $\begin{array}{c}-765.643099 \\
(0.051346)\end{array}$ & $\begin{array}{c}-765.642636 \\
(0.051513)\end{array}$ & 1.22 & & 5.49 & $\begin{array}{c}5.89 \\
(58.4)\end{array}$ & 1.36 \\
\hline $\begin{array}{l}\text { DFT/B3LYP/ } \\
6-311++\mathrm{G}(\mathrm{d}, \mathrm{p})\end{array}$ & $\begin{array}{c}-767.175861 \\
(0.050625)\end{array}$ & $\begin{array}{c}-767.174855 \\
(0.050591)\end{array}$ & 2.64 & & 6.09 & $\begin{array}{c}6.49 \\
(68.2)\end{array}$ & 1.34 \\
\hline \multicolumn{8}{|c|}{ Isocyano(isocyanomethyl)silane } \\
\hline MP2/6-311++G(d,p) & $\begin{array}{l}-514.604265 \\
(0.061471)\end{array}$ & $\begin{array}{c}-514.603668 \\
(0.061624)\end{array}$ & 1.57 & & 1.63 & $\begin{array}{c}2.56 \\
(60.4)\end{array}$ & 3.12 \\
\hline $\begin{array}{l}\text { DFT/B3LYP/ } \\
6-311++\mathrm{G}(\mathrm{d}, \mathrm{p})\end{array}$ & $\begin{array}{c}-515.745887 \\
(0.060865)\end{array}$ & $\begin{array}{c}-515.744528 \\
(0.060880)\end{array}$ & 3.57 & & 3.43 & $\begin{array}{c}3.61 \\
(58.4)\end{array}$ & 0.60 \\
\hline $\begin{array}{l}\text { MP2/ } \\
6-311+G(3 d f, 2 p)\end{array}$ & -514.754754 & -514.753655 & 2.88 & & & & \\
\hline G2MP2 & $\begin{array}{c}-514.851786 \\
(0.058859)\end{array}$ & $\begin{array}{c}-514.850637 \\
(0.058900)\end{array}$ & & 3.02 & 2.84 & $\begin{array}{c}2.94 \\
(52.3)\end{array}$ & 0.32 \\
\hline
\end{tabular}

* Values in bracket are zero point energies in Hartrees; ** Values in bracket are percentage of trans rotamers in the equilibrium mixture at $298.15 \mathrm{~K}$.

hydrogen atom. Further, the bond lengths of the transition state structures are comparable to those of the trans and gauche rotamers.

Table 2 summarises the energies of the trans and gauche rotamers, $\Delta \mathrm{E}$ and related thermodynamical parameters. Some conclusions can be drawn from Table 2. Firstly, for all the three molecules, the trans rotamer has lower energy than the gauche form. Secondly, $\triangle \mathrm{E}$ is generally larger from DFT than MP2 computations when the same basis set is used. Lastly, the percentage of the trans rotamer can be calculated using the free energy difference $(\Delta \mathrm{G})$ between the trans and gauche rotamers and the fact that there are two equivalent gauche forms. It is found that at $298.15 \mathrm{~K}$, in general, the equilibrium mixture is more populated with the trans conformer and this is in agreement with literature for 1,2-diisocyanoethane [13]. The rotational barriers, the energy difference between the gauche rotamer and transition state, for 1,2-diisocyanoethane, 1,2-diisocyanodisilane and isocyano(isocyanomethyl)silane are 16.32, 6.59 and 2.32 $\mathrm{kJ} / \mathrm{mol}$ (MP2) and 11.35, 4.46 and $1.77 \mathrm{~kJ} / \mathrm{mol}$ (DFT) respectively. It can be found that the rotational barrier decreases and it becomes comparable to the energy difference for isocyano(isocyanomethyl)silane. Apart from these, for a given method, the relative energy indicates that a dicyano compound has lower energy than its isomeric diisocyano compound [11]. 
Table 3. Vibrational analysis of the trans and gauche rotamers 1,2-diisocyanoethane

\begin{tabular}{|c|c|c|c|c|c|c|c|c|c|c|}
\hline & & Trans & & & & & Gauche & & & \\
\hline Vibration & MP2 & DFT & Experimental & Mode & Assignment & MP2 & DFT & Experimental & Mode & Assignmer \\
\hline$v_{1}$ & $\begin{array}{l}3115.4 \\
(0)\end{array}$ & $\begin{array}{l}3058.2 \\
(0)\end{array}$ & 2990 & $\overline{A_{g}}$ & C-H stretch & $\begin{array}{l}3170.9 \\
(1.9) \\
\end{array}$ & $\begin{array}{l}3095.4 \\
(4.2)\end{array}$ & 2990 & $\bar{A}$ & C-H stretch \\
\hline$v_{2}$ & $\begin{array}{l}2134.4 \\
(0)\end{array}$ & $\begin{array}{l}2219.9 \\
(0)\end{array}$ & 2155 & & $\mathrm{~N} \equiv \mathrm{C}$ stretch & $\begin{array}{l}3113.0 \\
(15.5)\end{array}$ & $\begin{array}{l}3057.5 \\
(18.5)\end{array}$ & 2958 & & C-H stretch \\
\hline$v_{3}$ & $\begin{array}{l}1513.1 \\
(0)\end{array}$ & $\begin{array}{l}1494.4 \\
(0)\end{array}$ & 1445 & & $\mathrm{CH}_{2}$ scissors & $\begin{array}{l}2134.0 \\
(122.9)\end{array}$ & $\begin{array}{l}2219.9 \\
(184.5)\end{array}$ & 2155 & & $\mathrm{~N} \equiv \mathrm{C}$ stretch \\
\hline$v_{4}$ & $\begin{array}{l}1428.5 \\
(0)\end{array}$ & $\begin{array}{l}1403.7 \\
(0)\end{array}$ & & & $\mathrm{CH}_{2}$ wag & $\begin{array}{l}1508.1 \\
(0.05) \\
\end{array}$ & $\begin{array}{l}1486.7 \\
(0.1)\end{array}$ & 1445 & & $\mathrm{CH}_{2}$ scissors \\
\hline$v_{5}$ & $\begin{array}{l}1079.4 \\
(0)\end{array}$ & $\begin{array}{l}1036.5 \\
(0)\end{array}$ & & & C-C stretch & $\begin{array}{l}1415.3 \\
(12.4)\end{array}$ & $\begin{array}{l}1388.4 \\
(12.7)\end{array}$ & 1358 & & $\mathrm{CH}_{2}$ wag \\
\hline$v_{6}$ & $\begin{array}{l}1017.1 \\
(0)\end{array}$ & $\begin{array}{l}996.7 \\
(0)\end{array}$ & 1002 & & C-NC stretch & $\begin{array}{l}1316.8 \\
(0.7)\end{array}$ & $\begin{array}{l}1308.3 \\
(0.5)\end{array}$ & 1275 & & $\mathrm{CH}_{2}$ twist \\
\hline$v_{7}$ & $\begin{array}{l}469.4 \\
(0) \\
\end{array}$ & $\begin{array}{l}459.1 \\
(0) \\
\end{array}$ & 520 & & C-CN bend & $\begin{array}{l}1112.7 \\
(0.02) \\
\end{array}$ & $\begin{array}{l}1086.6 \\
(0.5)\end{array}$ & 1073 & & C-C stretch \\
\hline$v_{8}$ & $\begin{array}{l}186.0 \\
(0)\end{array}$ & $\begin{array}{l}196.3 \\
(0)\end{array}$ & 209 & & C-N-C bend & $\begin{array}{l}1066.9 \\
(8.6)\end{array}$ & $\begin{array}{l}1037.3 \\
(7.7)\end{array}$ & 1002 & & $\mathrm{CH}_{2}$ rock \\
\hline$v_{9}$ & $\begin{array}{l}3192.2 \\
(1.8) \\
\end{array}$ & $\begin{array}{l}3117.5 \\
(5.3) \\
\end{array}$ & 2998 & $\mathrm{~A}_{u}$ & C-H stretch & $\begin{array}{l}851.8 \\
(11.1) \\
\end{array}$ & $\begin{array}{l}821.1 \\
(8.6) \\
\end{array}$ & 823 & & C-NC stretch \\
\hline$v_{10}$ & $\begin{array}{l}1268.5 \\
(4.1) \\
\end{array}$ & $\begin{array}{l}1258.0 \\
(3.1) \\
\end{array}$ & 1231 & & $\mathrm{CH}_{2}$ twist & $\begin{array}{l}395.6 \\
(0.4) \\
\end{array}$ & $\begin{array}{l}389.3 \\
(0.3) \\
\end{array}$ & 395 & & C-C-N bend \\
\hline$v_{11}$ & $\begin{array}{l}804.5 \\
(0.03) \\
\end{array}$ & $\begin{array}{l}794.6 \\
(2.8) \\
\end{array}$ & 778 & & $\mathrm{CH}_{2} \mathrm{rock}$ & $\begin{array}{l}304.4 \\
(0.6) \\
\end{array}$ & $\begin{array}{l}299.7 \\
(0.9) \\
\end{array}$ & 308 & & C-N-C bend \\
\hline$v_{12}$ & $\begin{array}{l}280.6 \\
(2.8)\end{array}$ & $\begin{array}{l}297.9 \\
(4.1)\end{array}$ & 306 & & C-N-C bend & $\begin{array}{l}167.7 \\
(0.6)\end{array}$ & $\begin{array}{l}173.4 \\
(0.6)\end{array}$ & 184 & & C-N-C bend \\
\hline$v_{13}$ & $\begin{array}{l}82.5 \\
(23.1) \\
\end{array}$ & $\begin{array}{l}84.1 \\
(20.4) \\
\end{array}$ & & & torsion & $\begin{array}{l}86.2 \\
(4.4) \\
\end{array}$ & $\begin{array}{l}82.2 \\
(4.6) \\
\end{array}$ & 108 & & torsion \\
\hline$v_{14}$ & $\begin{array}{l}3154.0 \\
(0)\end{array}$ & $\begin{array}{l}3096.1 \\
(0)\end{array}$ & 2958 & $\mathrm{~B}_{\mathrm{g}}$ & C-H stretch & $\begin{array}{l}3180.9 \\
(2.8)\end{array}$ & $\begin{array}{l}3106.1 \\
(6.1)\end{array}$ & 2990 & B & C-H stretch \\
\hline$v_{15}$ & $\begin{array}{l}1326.9 \\
(0)\end{array}$ & $\begin{array}{l}1323.8 \\
(0)\end{array}$ & 1022 & & $\mathrm{CH}_{2}$ twist & $\begin{array}{l}3111.3 \\
(2.7)\end{array}$ & $\begin{array}{l}3054.1 \\
(2.9)\end{array}$ & 2958 & & C-H stretch \\
\hline$v_{16}$ & $\begin{array}{l}1043.1 \\
(0) \\
\end{array}$ & $\begin{array}{l}1108.5 \\
(0)\end{array}$ & & & $\mathrm{CH}_{2}$ rock & $\begin{array}{l}2136.5 \\
(100.5) \\
\end{array}$ & $\begin{array}{l}2217.6 \\
(183.3)\end{array}$ & 2180 & & $\mathrm{~N} \equiv C$ stretch \\
\hline$v_{17}$ & $\begin{array}{l}336.1 \\
(0) \\
\end{array}$ & $\begin{array}{l}265.1 \\
(0) \\
\end{array}$ & 274 & & C-N-C bend & $\begin{array}{l}1508.5 \\
(18.0) \\
\end{array}$ & $\begin{array}{l}1487.2 \\
(19.6)\end{array}$ & 1445 & & $\mathrm{CH}_{2}$ scissors \\
\hline$v_{18}$ & $\begin{array}{l}3123.7 \\
(12.7)\end{array}$ & $\begin{array}{l}3067.2 \\
(14.4)\end{array}$ & 2958 & $\mathrm{~B}_{u}$ & C-H stretch & $\begin{array}{l}1402.2 \\
(19.4)\end{array}$ & $\begin{array}{l}1390.1 \\
(22.2\end{array}$ & 1344 & & $\mathrm{CH}_{2}$ wag \\
\hline$v_{19}$ & $\begin{array}{l}2137.2 \\
(242.4)\end{array}$ & $\begin{array}{l}2217.6 \\
(396.7)\end{array}$ & 2155 & & $\mathrm{~N} \equiv \mathrm{C}$ stretch & $\begin{array}{l}1285.3 \\
(3.4)\end{array}$ & $\begin{array}{l}1270.8 \\
(2.5)\end{array}$ & 1248 & & $\mathrm{CH}_{2}$ twist \\
\hline$v_{20}$ & $\begin{array}{l}1517.8 \\
(10.8)\end{array}$ & $\begin{array}{l}1503.3 \\
(11.9)\end{array}$ & 1459 & & $\mathrm{CH}_{2}$ scissors & $\begin{array}{l}1047.7 \\
(4.6)\end{array}$ & $\begin{array}{l}1029.1 \\
(5.0)\end{array}$ & 1022 & & C-C stretch \\
\hline$v_{21}$ & $\begin{array}{l}1334.8 \\
(21.5) \\
\end{array}$ & $\begin{array}{l}1336.6 \\
(22.1) \\
\end{array}$ & 1344 & & $\mathrm{CH}_{2}$ wag & $\begin{array}{l}868.5 \\
(12.2) \\
\end{array}$ & $\begin{array}{l}855.9 \\
(12.7) \\
\end{array}$ & 834 & & $\mathrm{CH}_{2}$ rock \\
\hline$v_{22}$ & $\begin{array}{l}986.0 \\
(43.7)\end{array}$ & $\begin{array}{l}965.6 \\
(38.2)\end{array}$ & 945 & & C-NC stretch & $\begin{array}{l}565.2 \\
(18.7)\end{array}$ & $\begin{array}{l}550.7 \\
(20.1)\end{array}$ & 558 & & C-C-N bend \\
\hline$v_{23}$ & $\begin{array}{l}416.2 \\
(13.5) \\
\end{array}$ & $\begin{array}{l}416.9 \\
(16.1) \\
\end{array}$ & 520 & & C-CN bend & $\begin{array}{l}255.5 \\
(0.5) \\
\end{array}$ & $\begin{array}{l}263.4 \\
(1.0) \\
\end{array}$ & 274 & & C-N-C bend \\
\hline$v_{24}$ & $\begin{array}{l}118.9 \\
(19.5)\end{array}$ & $\begin{array}{l}126.4 \\
(16.1)\end{array}$ & 187 & & C-N-C bend & $\begin{array}{l}191.5 \\
(3.6)\end{array}$ & $\begin{array}{l}196.3 \\
(2.1)\end{array}$ & 207 & & C-N-C bend \\
\hline
\end{tabular}

Frequencies are in $\left(\mathrm{cm}^{-1}\right)$, values in bracket are intensities in $\left(\mathrm{km} \mathrm{mol}^{-1}\right)$, experimental frequencies are from Ref [12] 
Table 4. Vibrational analysis of the trans and gauche rotamers of 1,2-diisocyanodisilane

\begin{tabular}{|c|c|c|c|c|c|c|c|c|}
\hline & & Trans & & & & Gauche & & \\
\hline Vibration & MP2 & DFT & Mode & Assignment & MP2 & DFT & Mode & Assignment \\
\hline$v_{1}$ & $\begin{array}{l}2345.5 \\
(0) \\
\end{array}$ & $\begin{array}{l}2260.4 \\
(0)\end{array}$ & $\mathrm{A}_{\mathrm{g}}$ & Si-H stretch & $\begin{array}{l}2351.9 \\
(14.5) \\
\end{array}$ & $\begin{array}{l}2264.6 \\
(12.6) \\
\end{array}$ & A & $\mathrm{Si}-\mathrm{H}$ stretch \\
\hline$v_{2}$ & $\begin{array}{l}2083.9 \\
(0) \\
\end{array}$ & $\begin{array}{l}2148.9 \\
(0)\end{array}$ & & $\mathrm{N} \equiv \mathrm{C}$ stretch & $\begin{array}{l}2341.0 \\
(88.2)\end{array}$ & $\begin{array}{l}2254.5 \\
(71.8) \\
\end{array}$ & & Si-H stretch \\
\hline$v_{3}$ & $\begin{array}{l}983.8 \\
(0) \\
\end{array}$ & $\begin{array}{l}947.7 \\
(0)\end{array}$ & & $\mathrm{SiH}_{2}$ scissors & $\begin{array}{l}2084.4 \\
(292.5)\end{array}$ & $\begin{array}{l}2149.4 \\
(406.7) \\
\end{array}$ & & $\mathrm{N} \equiv \mathrm{C}$ stretch \\
\hline$v_{4}$ & $\begin{array}{l}894.3 \\
(0)\end{array}$ & $\begin{array}{l}870.1 \\
(0)\end{array}$ & & $\mathrm{SiH}_{2}$ wag & $\begin{array}{l}986.8 \\
(76.1)\end{array}$ & \begin{tabular}{|l}
949.4 \\
$(74.8)$ \\
\end{tabular} & & $\mathrm{SiH}_{2}$ scissors \\
\hline$v_{5}$ & $\begin{array}{l}\begin{array}{l}659.4 \\
(0)\end{array} \\
\end{array}$ & $\begin{array}{l}655.5 \\
(0)\end{array}$ & & Si-N stretch & $\begin{array}{l}889.8 \\
(125.0)\end{array}$ & $\begin{array}{l}861.9 \\
(83.4) \\
\end{array}$ & & $\mathrm{SiH}_{2}$ wag \\
\hline$v_{6}$ & $\begin{array}{l}473.6 \\
(0) \\
\end{array}$ & $\begin{array}{l}454.3 \\
(0) \\
\end{array}$ & & Si-Si stretch & $\begin{array}{l}755.0 \\
(7.3) \\
\end{array}$ & $\begin{array}{l}739.4 \\
(8.8) \\
\end{array}$ & & $\mathrm{SiH}_{2}$ twist \\
\hline$v_{7}$ & $\begin{array}{l}266.1 \\
(0)\end{array}$ & $\begin{array}{l}268.7 \\
(0)\end{array}$ & & Si-Si-N bend & $\begin{array}{l}674.1 \\
(73.8) \\
\end{array}$ & $\begin{array}{l}669.8 \\
(57.6) \\
\end{array}$ & & Si-N stretch \\
\hline$v_{8}$ & $\begin{array}{l}112.8 \\
(0)\end{array}$ & $\begin{array}{l}122.2 \\
(0)\end{array}$ & & $\mathrm{Si}-\mathrm{N} \equiv \mathrm{C}$ bend & $\begin{array}{l}570.3 \\
(13.4)\end{array}$ & $\begin{array}{l}543.0 \\
(9.7)\end{array}$ & & $\mathrm{SiH}_{2}$ rock \\
\hline$v_{9}$ & $\begin{array}{l}2361.9 \\
(131.1)\end{array}$ & $\begin{array}{l}2275.5 \\
(128.9\end{array}$ & $\overline{A_{u}}$ & Si-H stretch & $\begin{array}{l}431.5 \\
(13.9) \\
\end{array}$ & \begin{tabular}{|l}
401.1 \\
$(12.7)$ \\
\end{tabular} & & Si-Si stretch \\
\hline$v_{10}$ & $\begin{array}{l}735.1 \\
(48.3) \\
\end{array}$ & $\begin{array}{l}726.0 \\
(43.0) \\
\end{array}$ & & $\mathrm{SiH}_{2}$ twist & $\begin{array}{l}222.1 \\
(1.8) \\
\end{array}$ & $\begin{array}{l}235.1 \\
(1.4) \\
\end{array}$ & & Si-Si-N bend \\
\hline$v_{11}$ & $\begin{array}{l}376.2 \\
(27.3) \\
\end{array}$ & $\begin{array}{l}368.1 \\
(22.7) \\
\end{array}$ & & $\mathrm{SiH}_{2}$ rock & $\begin{array}{l}186.7 \\
(0.1) \\
\end{array}$ & $\begin{array}{l}201.7 \\
(0.1) \\
\end{array}$ & & $\mathrm{Si}-\mathrm{N} \equiv \mathrm{C}$ bend \\
\hline$v_{12}$ & $\begin{array}{l}180.2 \\
(0.4)\end{array}$ & $\begin{array}{l}201.7 \\
(0.1) \\
\end{array}$ & & $\mathrm{Si}-\mathrm{N} \equiv \mathrm{C}$ bend & $\begin{array}{l}77.3 \\
0.08 \\
\end{array}$ & $\begin{array}{l}82.0 \\
(0.3)\end{array}$ & & $\mathrm{Si}-\mathrm{N} \equiv \mathrm{C}$ bend \\
\hline$v_{13}$ & $\begin{array}{l}25.8 \\
(12.0)\end{array}$ & $\begin{array}{l}32.9 \\
(10.0)\end{array}$ & & torsion & $\begin{array}{l}36.6 \\
(3.3)\end{array}$ & $\begin{array}{l}33.9 \\
(3.2)\end{array}$ & & torsion \\
\hline$v_{14}$ & $\begin{array}{l}2354.3 \\
(0) \\
\end{array}$ & $\begin{array}{l}2267.1 \\
(0)\end{array}$ & $\mathrm{B}_{\mathrm{g}}$ & Si-H stretch & $\begin{array}{l}2356.3 \\
(115.4) \\
\end{array}$ & $\begin{array}{l}2269.1 \\
(109.6) \\
\end{array}$ & B & Si-H stretch \\
\hline$v_{15}$ & $\begin{array}{l}754.0 \\
(0) \\
\end{array}$ & $\begin{array}{l}740.2 \\
(0) \\
\end{array}$ & & $\mathrm{SiH}_{2}$ twist & $\begin{array}{l}2333.9 \\
(48.2) \\
\end{array}$ & $\begin{array}{l}2245.3 \\
(46.5) \\
\end{array}$ & & Si-H stretch \\
\hline$v_{16}$ & $\begin{array}{l}608.0 \\
(0) \\
\end{array}$ & $\begin{array}{l}592.3 \\
(0) \\
\end{array}$ & & $\mathrm{SiH}_{2}$ rock & $\begin{array}{l}2082.2 \\
(187.8) \\
\end{array}$ & $\begin{array}{l}2144.5 \\
(321.0) \\
\end{array}$ & & $\mathrm{N} \equiv \mathrm{C}$ stretch \\
\hline$v_{17}$ & $\begin{array}{l}176.3 \\
(0)\end{array}$ & $\begin{array}{l}\begin{array}{l}197.1 \\
(0)\end{array} \\
\end{array}$ & & $\mathrm{Si}-\mathrm{N} \equiv \mathrm{C}$ bend & $\begin{array}{l}963.8 \\
(115.6)\end{array}$ & $\begin{array}{l}928.6 \\
(85.3) \\
\end{array}$ & & $\mathrm{SiH}_{2}$ scissors \\
\hline$v_{18}$ & $\begin{array}{l}2342.8 \\
(99.9) \\
\end{array}$ & $\begin{array}{l}2256.8 \\
(91.6) \\
\end{array}$ & $\mathrm{B}_{\mathrm{u}}$ & Si-H stretch & $\begin{array}{l}837.5 \\
(487.9) \\
\end{array}$ & $\begin{array}{l}811.1 \\
(479.3) \\
\end{array}$ & & $\mathrm{SiH}_{2}$ wag \\
\hline$v_{19}$ & $\begin{array}{l}2082.0 \\
(506.2)\end{array}$ & $\begin{array}{l}2144.5 \\
(747.0)\end{array}$ & & $\mathrm{N} \equiv \mathrm{C}$ stretch & $\begin{array}{l}744.2 \\
(31.2)\end{array}$ & $\begin{array}{l}731.1 \\
(29.7)\end{array}$ & & $\mathrm{SiH}_{2}$ twist \\
\hline$v_{20}$ & $\begin{array}{l}972.0 \\
(220.6) \\
\end{array}$ & $\begin{array}{l}943.4 \\
(182.8)\end{array}$ & & $\mathrm{SiH}_{2}$ scissors & $\begin{array}{l}659.9 \\
(64.7) \\
\end{array}$ & $\begin{array}{l}656.5 \\
(72.2) \\
\end{array}$ & & Si-N stretch \\
\hline$v_{21}$ & $\begin{array}{l}802.6 \\
(594.3)\end{array}$ & $\begin{array}{l}784.2 \\
(527.5)\end{array}$ & & $\mathrm{SiH}_{2}$ wag & $\begin{array}{l}431.5 \\
(13.9) \\
\end{array}$ & $\begin{array}{l}453.6 \\
(42.8) \\
\end{array}$ & & $\mathrm{SiH}_{2}$ rock \\
\hline$v_{22}$ & $\begin{array}{l}658.7 \\
(233.1) \\
\end{array}$ & $\begin{array}{l}654.2 \\
(215.9)\end{array}$ & & Si-N stretch & $\begin{array}{l}222.1 \\
(1.8) \\
\end{array}$ & $\begin{array}{l}269.2 \\
(9.1) \\
\end{array}$ & & Si-Si-N bend \\
\hline$v_{23}$ & $\begin{array}{l}227.4 \\
(3.7) \\
\end{array}$ & $\begin{array}{l}242.8 \\
(3.9) \\
\end{array}$ & & Si-Si-N bend & $\begin{array}{l}186.7 \\
(0.1) \\
\end{array}$ & $\begin{array}{l}195.7 \\
(0.3) \\
\end{array}$ & & $\mathrm{Si}-\mathrm{N} \equiv \mathrm{C}$ bend \\
\hline$v_{24}$ & $\begin{array}{l}61.5 \\
(16.1) \\
\end{array}$ & $\begin{array}{l}67.4 \\
(13.3)\end{array}$ & & $\mathrm{Si}-\mathrm{N} \equiv \mathrm{C}$ bend & $\begin{array}{l}111.5 \\
(9.9\end{array}$ & $\begin{array}{l}116.5 \\
(8.1)\end{array}$ & & $\mathrm{Si}-\mathrm{N} \equiv \mathrm{C}$ bend \\
\hline
\end{tabular}

Frequencies are in $\left(\mathrm{cm}^{-1}\right)$, values in bracket are intensities in $\left(\mathrm{km} \mathrm{mol}^{-1}\right)$

The calculated infrared raw vibrational frequencies, their intensities and assignments of the trans and gauche rotamers of the title compounds are reported in Tables 3-5 respectively. The experimental infrared vibrational frequencies of the trans and gauche 
Table 5. Vibrational analysis of the trans and gauche rotamers of isocyano(isocyanomethyl)silane

\begin{tabular}{|c|c|c|c|c|c|c|c|c|}
\hline & & Trans & & & & Gauche & & \\
\hline Vibration & MP2 & DFT & Mode & Assignment & MP2 & DFT & Mode & Assignment \\
\hline$v_{1}$ & $\begin{array}{l}3165.6 \\
(0.004)\end{array}$ & $\begin{array}{l}3091.7 \\
(0.4)\end{array}$ & $\mathrm{A}^{\prime \prime}$ & C-H stretch & $\begin{array}{l}3154.3 \\
(0.3)\end{array}$ & $\begin{array}{l}3078.1 \\
(1.1)\end{array}$ & A & C-H stretch \\
\hline$v_{2}$ & $\begin{array}{l}2380.8 \\
(74.8)\end{array}$ & $\begin{array}{l}2292.1 \\
(74.1)\end{array}$ & & Si-H stretch & $\begin{array}{l}3093.6 \\
(7.0)\end{array}$ & $\begin{array}{l}3033.7 \\
(8.3)\end{array}$ & & C-H stretch \\
\hline$v_{3}$ & $\begin{array}{l}1251.3 \\
(8.0) \\
\end{array}$ & $\begin{array}{l}1242.7 \\
(6.6) \\
\end{array}$ & & $\mathrm{CH}_{2}$ twist & $\begin{array}{l}2376.4 \\
(67.8) \\
\end{array}$ & $\begin{array}{l}2290.0 \\
(64.7)\end{array}$ & & Si-H stretch \\
\hline$v_{4}$ & $\begin{array}{l}869.9 \\
(22.4) \\
\end{array}$ & $\begin{array}{l}855.0 \\
(21.3) \\
\end{array}$ & & $\mathrm{CH}_{2}$ rock & $\begin{array}{l}2355.2 \\
(78.7) \\
\end{array}$ & $\begin{array}{l}2268.2 \\
(69.7) \\
\end{array}$ & & Si-H stretch \\
\hline$v_{5}$ & $\begin{array}{l}724.6 \\
(32.1) \\
\end{array}$ & $\begin{array}{l}713.0 \\
(30.1) \\
\end{array}$ & & $\mathrm{SiH}_{2}$ twist & $\begin{array}{l}2127.6 \\
(80.3)\end{array}$ & $\begin{array}{l}2211.8 \\
(152.4)\end{array}$ & & ${ }_{\mathrm{C}} \mathrm{N} \equiv \mathrm{C}$ stretch \\
\hline$v_{6}$ & $\begin{array}{l}507.0 \\
(14.9)\end{array}$ & $\begin{array}{l}502.7 \\
(13.4) \\
\end{array}$ & & $\mathrm{SiH}_{2}$ rock & $\begin{array}{l}2087.4 \\
(246.5)\end{array}$ & $\begin{array}{l}2150.1 \\
(363.3)\end{array}$ & & ${ }_{\mathrm{Si}} \mathrm{N} \equiv \mathrm{C}$ stretch \\
\hline$v_{7}$ & $\begin{array}{l}268.9 \\
(0.9) \\
\end{array}$ & $\begin{array}{l}279.7 \\
(1.5)\end{array}$ & & $\mathrm{C}-\mathrm{N} \equiv \mathrm{C}$ bend & $\begin{array}{l}1474.6 \\
(13.1)\end{array}$ & $\begin{array}{l}1460.3 \\
(13.3)\end{array}$ & & $\mathrm{CH}_{2}$ scissors \\
\hline$v_{8}$ & $\begin{array}{l}177.8 \\
(0.05)\end{array}$ & $\begin{array}{l}198.7 \\
(0.005)\end{array}$ & & $\mathrm{Si}-\mathrm{N} \equiv \mathrm{C}$ bend & $\begin{array}{l}1292.1 \\
(2.2)\end{array}$ & $\begin{array}{l}1271.5 \\
(1.2)\end{array}$ & & $\mathrm{CH}_{2}$ wag \\
\hline$v_{9}$ & $\begin{array}{l}52.7 \\
(18.3) \\
\end{array}$ & $\begin{array}{l}55.1 \\
(16.5) \\
\end{array}$ & & torsion & $\begin{array}{l}1258.9 \\
(14.7)\end{array}$ & $\begin{array}{l}1250.8 \\
(12.4) \\
\end{array}$ & & $\mathrm{CH}_{2}$ twist \\
\hline$v_{10}$ & $\begin{array}{l}3103.6 \\
(5.8)\end{array}$ & $\begin{array}{l}3046.6 \\
(5.9\end{array}$ & $\mathrm{A}^{\prime}$ & C-H stretch & $\begin{array}{l}993.1 \\
(13.1)\end{array}$ & $\begin{array}{l}976.7 \\
(2.3) \\
\end{array}$ & & C-N stretch \\
\hline$v_{11}$ & $\begin{array}{l}2366.5 \\
(43.6) \\
\end{array}$ & $\begin{array}{l}2279.2 \\
(41.3 \\
\end{array}$ & & Si-H stretch & $\begin{array}{l}985.1 \\
(125.4) \\
\end{array}$ & $\begin{array}{l}950.4 \\
(113.1)\end{array}$ & & $\mathrm{SiH}_{2}$ scissors \\
\hline$v_{12}$ & $\begin{array}{l}2125.3 \\
(83.7)\end{array}$ & $\begin{array}{l}2209.2 \\
(137.0)\end{array}$ & & ${ }_{\mathrm{C}} \mathrm{N} \equiv \mathrm{C}$ stretch & $\begin{array}{l}921.6 \\
(278.5)\end{array}$ & $\begin{array}{l}900.4 \\
(261.3)\end{array}$ & & $\mathrm{SiH}_{2}$ wag \\
\hline$v_{13}$ & $\begin{array}{l}2087.0 \\
(268.4)\end{array}$ & $\begin{array}{l}2150.2 \\
(405.5)\end{array}$ & & ${ }_{\mathrm{Si}} \mathrm{N} \equiv \mathrm{C}$ stretch & $\begin{array}{l}814.0 \\
(24.8)\end{array}$ & $\begin{array}{l}794.1 \\
(22.3)\end{array}$ & & $\mathrm{SiH}_{2}$ twist \\
\hline$v_{14}$ & $\begin{array}{l}1476.2 \\
(9.8)\end{array}$ & $\begin{array}{l}1465.3 \\
(9.9)\end{array}$ & & $\mathrm{CH}_{2}$ scissors & $\begin{array}{l}742.1 \\
(26.4)\end{array}$ & $\begin{array}{l}729.4 \\
(24.7)\end{array}$ & & $\mathrm{SiH}_{2}$ twist \\
\hline$v_{15}$ & $\begin{array}{l}1285.2 \\
(6.8)\end{array}$ & $\begin{array}{l}1265.3 \\
(4.3)\end{array}$ & & $\mathrm{CH}_{2}$ wag & $\begin{array}{l}714.0 \\
(24.6)\end{array}$ & $\begin{array}{l}701.7 \\
(28.3)\end{array}$ & & Si-C stretch \\
\hline$v_{16}$ & $\begin{array}{l}993.3 \\
(83.6) \\
\end{array}$ & $\begin{array}{l}968.4 \\
(30.8)\end{array}$ & & $\mathrm{SiH}_{2}$ scissors & $\begin{array}{l}547.5 \\
(72.0)\end{array}$ & $\begin{array}{l}635.8 \\
(62.4)\end{array}$ & & Si-N stretch \\
\hline$v_{17}$ & $\begin{array}{l}978.8 \\
(28.1) \\
\end{array}$ & $\begin{array}{l}956.7 \\
(64.4)\end{array}$ & & $\mathrm{SiH}_{2}$ scissors & $\begin{array}{l}575.5 \\
(42.9)\end{array}$ & $\begin{array}{l}556.9 \\
(35.6)\end{array}$ & & $\mathrm{SiH}_{2}$ rock \\
\hline$v_{18}$ & $\begin{array}{l}880.8 \\
(335.7)\end{array}$ & $\begin{array}{l}864.6 \\
(301.2)\end{array}$ & & $\mathrm{SiH}_{2}$ wag & $\begin{array}{l}380.8 \\
(11.7)\end{array}$ & $\begin{array}{l}378.4 \\
(12.1)\end{array}$ & & S-C-N bend \\
\hline$v_{19}$ & $\begin{array}{l}737.8 \\
(19.8) \\
\end{array}$ & $\begin{array}{l}711.6 \\
(18.3) \\
\end{array}$ & & Si-C stretch & $\begin{array}{l}282.5 \\
(0.9) \\
\end{array}$ & $\begin{array}{l}283.1 \\
(0.9) \\
\end{array}$ & & $\mathrm{C}-\mathrm{N} \equiv \mathrm{C}$ bend \\
\hline$v_{20}$ & $\begin{array}{l}669.4 \\
(100.0)\end{array}$ & $\begin{array}{l}664.4 \\
(87.0)\end{array}$ & & Si-N stretch & $\begin{array}{l}267.7 \\
(2.1)\end{array}$ & $\begin{array}{l}273.1 \\
(2.5)\end{array}$ & & $\mathrm{C}-\mathrm{N} \equiv \mathrm{C}$ bend \\
\hline$v_{21}$ & $\begin{array}{l}375.3 \\
(1.3) \\
\end{array}$ & $\begin{array}{l}375.2 \\
(2.1) \\
\end{array}$ & & $\mathrm{C}-\mathrm{N} \equiv \mathrm{C}$ bend & $\begin{array}{l}186.2 \\
(0.4) \\
\end{array}$ & $\begin{array}{l}198.2 \\
(0.1) \\
\end{array}$ & & $\mathrm{Si}-\mathrm{N} \equiv \mathrm{C}$ bend \\
\hline$v_{22}$ & $\begin{array}{l}275.0 \\
(5.5)\end{array}$ & $\begin{array}{l}281.6 \\
(6.1)\end{array}$ & & $\mathrm{Si}-\mathrm{N} \equiv \mathrm{C}$ bend & $\begin{array}{l}149.6 \\
(7.3)\end{array}$ & $\begin{array}{l}156.8 \\
(5.6)\end{array}$ & & $\mathrm{C}-\mathrm{N} \equiv \mathrm{C}$ bend \\
\hline$v_{23}$ & $\begin{array}{l}148.0 \\
(0.7)\end{array}$ & $\begin{array}{l}158.6 \\
(0.5)\end{array}$ & & $\mathrm{C}-\mathrm{N} \equiv \mathrm{C}$ bend & $\begin{array}{l}114.2 \\
(0.6)\end{array}$ & $\begin{array}{l}120.7 \\
(0.5)\end{array}$ & & $\mathrm{Si}-\mathrm{N} \equiv \mathrm{C}$ bend \\
\hline$v_{24}$ & $\begin{array}{l}81.9 \\
(18.7)\end{array}$ & $\begin{array}{l}89.2 \\
(15.9)\end{array}$ & & $\mathrm{Si}-\mathrm{N} \equiv \mathrm{C}$ bend & $\begin{array}{l}57.8 \\
(4.6)\end{array}$ & $\begin{array}{l}53.2 \\
(4.8)\end{array}$ & & torsion \\
\hline
\end{tabular}

Frequencies are in $\left(\mathrm{cm}^{-1}\right)$, values in bracket are intensities in $\left(\mathrm{km} \mathrm{mol}^{-1}\right)$

rotamers of 1,2-diisocyanoethane obtained in the liquid phase are also included in Table 3. The 24 modes of vibrations account for the irreducible representations $\Gamma_{\mathrm{v}}=8 \mathrm{~A}_{\mathrm{g}}+5 \mathrm{~A}_{\mathrm{u}}$ $+4 \mathrm{~B}_{\mathrm{g}}+7 \mathrm{~B}_{\mathrm{u}}$ of the $C_{2 \mathrm{~h}}$ point group of the trans rotamer of1,2-diisocyanoethane and 
1,2-diisocyanodisilane and $\Gamma_{\mathrm{v}}=13 \mathrm{~A}+11 \mathrm{~B}$ of the $C_{2}$ point group of the gauche rotamer of 1,2-diisocyanoethane and 1,2-diisocyanodisilane. Further, the 24 modes of vibrations account for the irreducible representations $\Gamma_{\mathrm{v}}=9 \mathrm{~A}^{\prime \prime}+15 \mathrm{~A}^{\prime}$ of the $C_{\mathrm{s}}$ point group of the trans rotamer of isocyano(isocyanomethyl)silane and $\Gamma_{\mathrm{v}}=24 \mathrm{~A}$ of the $C_{1}$ point group of the gauche rotamer of isocyano(isocyanomethyl)silane. These infrared vibrational frequencies are dominated by the high intensity of the $\mathrm{N} \equiv \mathrm{C}$ stretching frequency. It is interesting to note the close agreement of the calculated infrared raw vibrational frequencies (gas phase) and experimental literature data (liquid phase) [12] for 1,2diisocyanoethane. Apart from these, it can be found that substitution of the cyano groups by the isocyano groups affects mainly the vibrational frequencies where the cyano groups are attached [11].

\section{Conclusion}

This paper reports the gas phase theoretical study of the trans and gauche rotamers of 1,2-diisocyanoethane, novel 1,2-diisocyanodisilane and isocyano(isocyanomethyl)silane in terms of optimised molecular structures and infrared raw vibrational frequencies. The rotational barrier and related thermodynamic parameters are also given. The trans rotamer is more stable than gauche rotamer. An interesting outcome of this work is that some of the calculated parameters for 1,2-diisocyanoethane compare satisfactorily with experimental literature data. More interestingly, although the changes in structure and energy are relatively small when carbon is substituted by silicon, data for the trans and gauche rotamers for the novel 1,2-diisocyanodisilane and isocyano(isocyanomethyl)silane could serve as a reliable set of reference as their literature is limited.

Acknowledgments. The author acknowledges facilities from the University of Mauritius. The author also thanks anonymous referees for their comments to improve the manuscript.

\section{References}

1. Radom, L., Baker, J., Gill, P.M.W., Nobes, R.H., Riggs, N.V.: A Theoretical Approach to Molecular Conformational Analysis. J. Mol. Struct. 126, 271-290 (1985)

2. Wiberg, K.B., Murko, M.A.: Rotational barriers. 2. Energies of Alkane Rotamers. An Examination of Gauche Interactions. J. Am. Chem. Soc. 110, 8029-8038 (1988)

3. Wiberg, K.B., Keith, T.A., Frisch, M.J., Murcko, M.: Solvent Effects on 1,2-Dihaloethane Gauche/Trans Ratios. J. Phys. Chem. 99, 9072-9079 (1995)

4. Ramasami, P.: Gauche and Trans Conformers of 1,2-Dihaloethanes: A Study by Ab initio and Density Functional Methods. Lecture Series on Computer and Computational Sciences 4, 732-734 (2005)

5. Ramasami, P.: Gas Phase Study of the Gauche and Trans Conformers of 1-Fluoro-2- Haloethanes $\mathrm{CH} 2 \mathrm{~F}-\mathrm{CH} 2 \mathrm{X}(\mathrm{X}=\mathrm{Cl}, \mathrm{Br}, \mathrm{I})$ by $\mathrm{Ab}$ initio and Density Functional Methods: $\mathrm{Ab}$ sence of Gauche Effect. In: Alexandrov, V.N., van Albada, G.D., Sloot, P.M.A., Dongarra, J. (eds.) ICCS 2006. LNCS, vol. 3993, pp. 153-160. Springer, Heidelberg (2006) 
6. Fitzgerald, W.E., Janz, G.J.: Vibrational Spectra and Molecular Structure of 1,2 Dicyanoethane. J. Mol. Spectrosc. 1, 49-60 (1957)

7. Walker, G.A., Bhatia, S.C., Hall Jr., J.H.: Theoretical Investigation of the Hydration Properties for the Trans and Gauche Rotamers of Succinonitrile. Int. J. Quan. Chem. 38, 85-92 (1990)

8. Fengler, O.I., Ruoff, A.: Vibrational Spectra of Succinonitrile and its $\left[1,4-{ }^{13} \mathrm{C} 2\right]-$, $[2,2,3,3-$ $\left.{ }^{2} \mathrm{H} 4\right]$ - and $\left[1,4-{ }^{13} \mathrm{C} 2-2,2,3,3-{ }^{2} \mathrm{H} 4\right]$-Isotopomers and a Force Field of Succinonitrile. Spectrochimica Acta 57, 105-117 (2001)

9. Fengler, O.I.: The Vibrational Spectra of $\left[{ }^{15} \mathrm{~N} 2\right]-$ Succinonitrile. Spectrochimica Acta A57, 1627-1634 (2001)

10. Umar, Y., Morsy, M.A.: Ab initio and DFT Studies of the Molecular Structures and Vibrational Spectra of Succinonitrile 66, 1133-1140 (2007)

11. Ramasami, P.: Gas Phase Study of the Trans and Gauche Rotamers of 1,2-Dicyanoethane, novel 1,2-Dicyanodisilane and Cyano(cyanomethyl)silane by Ab initio and Density Functional Methods. Spectrochimica Acta 68, 752-756 (2007)

12. Schrumpf, G., Martin, S.: Conformation and Vibrational Spectra of 1,2-Diisocyanoethane. J. Mol. Struc. 81, 155-165 (1982)

13. Schrumpf, G., Trætteberg, M., Bakken, P., Seip, R.: The Molecular Structure and Conformational Equilibrium of 1,2-Diisocyano-ethane Studied by Gas-phase Electron Diffraction. J. Mol. Struc. 197, 339-347 (1989)

14. Dartiguenave, M., Dartiguenave, Y., Guitard, A., Mari, A., Beauchamp, A.L.: Synthesis and Characterization of Copper, Manganese and Zinc Complexes of the Bidentate Diisocyano Ligands 2,5-Dimethyl 2,5-Diisocyanohexane (TMB) and 1,2-Diisocyanoethane(DHB). Polyhedron 8, 317-323 (1989)

15. Gross, H., König, G.M.: Terpenoids from Marine Organisms: Unique Structures and their Pharmacological Potential. Phytochem. Rev. 5, 115-141 (2006)

16. Frisch, M.J., et al.: Gaussian 03, Revision B04, Gaussian Inc., Wallingford, CT (2004)

17. GaussView, Version 3.09, R. Dennington II, T. Keith, J. Millam, K. Eppinnett, W.L. Hovell and R. Gilliland, Semichem, Inc., Shawnee Mission, KS (2003) 